\title{
Source and supply of terrestrial organic matter affects aquatic microbial metabolism
}

\author{
Jay T. Lennon ${ }^{1,2, *}$, Liza E. Pfaff ${ }^{1}$ \\ ${ }^{1}$ Department of Biological Sciences, Dartmouth College, Hanover, New Hampshire 03755-3576, USA \\ ${ }^{2}$ Present address: Department of Ecology \& Evolutionary Biology, Box G, Brown University, Providence, \\ Rhode Island 02912, USA
}

\begin{abstract}
Aquatic ecosystems are connected to their surrounding watersheds through inputs of terrestrial-derived dissolved organic matter (DOM). The assimilation of this allochthonous resource by recipient bacterioplankton has consequences for food webs and the biogeochemistry of aquatic ecosystems. We used laboratory batch experiments to examine how variation in the source and supply (i.e. concentration) of DOM affects the productivity, respiration and growth efficiency of heterotrophic lake bacterioplankton. We created 6 different DOM sources from soils beneath nearmonotypic tree stands in a temperate deciduous-coniferous forest. We then exposed freshwater microcosms containing a natural microbial community to a $1100 \mu \mathrm{M}$ supply gradient of each DOM source. Bacterial productivity (BP) and bacterial respiration (BR) increased linearly over the broad gradient, on average consuming $7 \%$ of the standing pool of dissolved organic carbon (DOC). Bacterial metabolism was also influenced by the chemical composition of the DOM source. Carbon-specific productivity declined exponentially with an increase in the carbon:phosphorus (C:P) ratio of the different DOM sources, consistent with the predictions of ecological stoichiometry. Together, our shortterm laboratory experiments quantitatively describe the metabolic responses of freshwater bacterioplankton to variation in the supply of terrestrial-derived DOM. Furthermore, our results suggest that dissolved organic phosphorus (DOP) content, which may be linked to the identity of terrestrial vegetation, is indicative of DOM quality and influences the productivity of freshwater bacterioplankton.
\end{abstract}

KEY WORDS: Allochthonous $\cdot$ Bacteria $\cdot$ DOC $\cdot$ DOM $\cdot$ Ecosystem $\cdot$ Plankton $\cdot$ Stoichiometry $\cdot$ Subsidy

\section{INTRODUCTION}

The metabolic activities of heterotrophic bacteria have important implications for the functioning of aquatic ecosystems. From a biogeochemical perspective, bacterial respiration (BR) constitutes a major carbon flux in freshwater and marine ecosystems (Biddanda et al. 2001, Gonzalez et al. 2003). From a foodweb perspective, bacterial productivity (BP) converts reduced carbon into biomass that can be ingested by protists and macrozooplankton (Langenheder \& Jürgens 2001, Sherr \& Sherr 2002). BP and BR are metabolic activities that are fueled primarily through the consumption of dissolved organic matter (DOM). In aquatic ecosystems, DOM is supplied to bacteria both locally by primary producers (Bertilsson \& Jones 2003) and externally by inputs from surrounding terrestrial ecosystems (Aitkenhead-Peterson et al. 2003).

Comparative studies have revealed that BP is positively correlated with net primary productivity (NPP) across fresh- and saltwater ecosystems (Cole et al. 1988), but is usually 70 to $90 \%$ less than NPP (Ducklow et al. 2002, Gaedke et al. 2002). This observation suggests that bacterial metabolism may be tightly coupled to the local production of photosynthetically derived DOM. Bacterial carbon demand, however, often exceeds NPP. For example, BR alone is greater than NPP in most oligotrophic waterbodies (del Giorgio et al. 1997) and BP:NPP ratios can sometimes exceed unity in freshwater ecosystems (Jansson et al. 2000, Karlsson et al. 2002, Waiser \& Robarts 2004). These results demonstrate that bacterial metabolism can be 
decoupled from local primary productivity. One potential explanation for this decoupling is that bacterial carbon demand is subsidized by terrestrial-derived DOM.

Terrestrial ecosystems export large quantities of DOM to inland, estuarine and coastal marine waterbodies (Goni et al. 1997, Findlay et al. 1998, Neff \& Asner 2001). However, terrestrial-derived DOM has traditionally been considered a poor-quality resource for aquatic bacteria because it is relatively old (Raymond \& Bauer 2001) and comprises humic compounds with low nutritional content (McKnight \& Aiken 1998). Nevertheless, a few lines of evidence indicate that bacterial metabolism may be supported to varying degrees by inputs of terrestrial DOM. First, numerous laboratory studies have directly demonstrated that aquatic bacteria grow on terrestrial fractions of DOM (Tranvik 1988, Bano et al. 1997, Moran \& Hodson 1994). Second, as lake DOM concentrations increase, community respiration tends to exceed local primary productivity (Hanson et al. 2003), suggesting that allochthonous carbon may be consumed by heterotrophic bacteria. Third, experimental isotope-enrichment of Wisconsin lakes revealed that 35 to $70 \%$ of the bacterial biomass may originate from terrestrialderived carbon (Kritzberg et al. 2004). Despite these studies, however, the relationship between aquatic bacterial metabolism and terrestrial carbon inputs remains unclear (Findlay 2003).

One potentially important factor that may influence aquatic bacterial metabolism is the bulk concentration of terrestrial DOM. In freshwater ecosystems, bulk DOM concentrations range over 2 orders of magnitude (Mulholland 2003) and can be explained in part by watershed attributes including wetland cover (Engstrom 1987, Gergel et al. 1999), soil properties (Nelson et al. 1993, McClain et al. 1997), drainage area (Rasmussen et al. 1989), forest cover (Goodale et al. 2000, Canham et al. 2004) and hydrology (Hinton et al. 1997, Boyer et al. 2000). Given the high demand for organic carbon by heterotrophic bacteria (Kirchman \& Rich 1997), BP and BR may be expected to increase along increasing DOM concentration gradients.

Aquatic bacterial metabolism is also affected by the chemical attributes of different DOM sources, including its oxidation state (Vallino et al. 1996), molecular weight (Weiss \& Simon 1999), humic content (Moran \& Hodson 1990), percentage of aliphatic compounds (Sun et al. 1997) and the stoichiometric ratios of growth-limiting nutrients (Hunt et al. 2000, Castillo et al. 2003). Most of the DOM in freshwater and coastal ecosystems originates from DOM in the soils of nearby terrestrial ecosystems (Thurman 1985). Soil DOM, which tends to be in equilibrium with solid-phase soil organic matter (Zsolnay 1996, Strobel et al. 2001), is influenced by a number of factors including physical properties, sorption and biodegradation (Qualls \& Haines 1992, Chantigny 2003). Soil DOM chemistry is also affected by the identity of the parent vegetation within the watershed (Grieve \& Marsden 2001). The chemistry of plant material differs among taxonomic groups (Likens \& Bormann 1970) and these differences are often reflected in the local soil organic matter (Preston \& Trofymow 2000, Finzi et al. 2001, Quideau et al. 2001) because of litterfall, root exudation and local microbial interactions (Kalbitz et al. 2000). As such, variation in the chemical attributes of DOM due to the heterogeneity of terrestrial ecosystems may in turn affect microbial metabolism in aquatic ecosystems.

This study examined how aquatic bacteria respond to variation in the source and supply of terrestrial DOM. We created different DOM sources from soils under different types of terrestrial vegetation. Then, using batch cultures, we measured BP, BR, bacterial growth efficiency (BGE) and rates of carbon consumption of a lake microbial community on varying concentrations (i.e. supply) of the different DOM sources. In general, we predicted that bacterial metabolism would increase with DOM concentration. However, we also predicted that the relationship between bacterial metabolism and DOM concentration would be modified by the chemical attributes of the different DOM sources. Using a combination of univariate and multivariate statistical approaches, we identified DOM chemical attributes that had a strong influence on aquatic microbial metabolism.

\section{MATERIALS AND METHODS}

Soil collection and DOM preparation. Through experimental leaching, we created 6 different terrestrial DOM sources from the organic $(\mathrm{Oa} / \mathrm{A})$ soil horizons underneath near-monoculture stands of some of the most common trees in New England forests: pine Pinus strobus, hemlock Tsuga canadensis, beech Fagus grandifolia, maple Acer saccharum, birch Betula alleghaniensis and B. populifolia, and oak Quercus rubra and $Q$. velutina. We collected soils from a total of 6 different sites ( $\leq 200 \mathrm{~m}$ from a stream or lake) located within $100 \mathrm{~km}$ of Hanover, New Hampshire, USA. The soils represented a mixture of inceptisols and entisols and, based on texture, were classified as fine sandy loams and sandy loams.

To create each DOM source, we dried the soils at $60^{\circ} \mathrm{C}$, pooled equal amounts of soil (100 g total) from multiple sites, and then extracted the organic matter for $48 \mathrm{~h}$ in $0.1 \mathrm{~N} \mathrm{NaOH}$ (Schnitzer 1982). We removed particulate material $(>0.7 \mu \mathrm{m})$ via serial filtration and then dialyzed (500 Da cellulose ester, Spectrum Labo- 
ratories) the leachates in distilled water buffers for $24 \mathrm{~h}$ to reduce concentrations of salts and inorganic nutrients (Vinebrooke \& Leavitt 1998, Lennon 2004a). We bottled the dialyzed leachates and had them gammairradiated (25 kGy dose, SteriGenics International) in order to kill soil-associated microorganisms while still maintaining the chemical integrity of the organic matter (McNamara et al. 2003).

Leachate characterization. We analyzed each DOM source for a suite of inorganic and organic attributes. We measured DOC with a Tekmar-Dohrmann TIC/ TOC analyzer after $\mathrm{H}_{2} \mathrm{SO}_{4}$ digestion, and measured total nitrogen (TN) and total phosphorus (TP) spectrophotometrically after persulfate digestion (APHA 1998). After the filtration and dialysis procedures, we assumed that TN and TP represented dissolved organic nitrogen (DON) and dissolved organic phosphorus (DOP), respectively. In addition, we measured 15 major elements with inductively coupled plasma (ICP) atomic emission spectroscopy (AES) on Spectro Analytical Model FTM-08 ICPOES (see Table 1). We measured polyphenolic compounds using the Prussian Blue assay (Price \& Bulter 1977) against a tannic acid standard (Herrera-Silveira \& Ramirez-Ramirez 1996, Thoss et al. 2002) and humic acids as the amount of precipitated DOC after acidifying the leachate to $\mathrm{pH} 2$ with $\mathrm{H}_{3} \mathrm{PO}_{4}$. We measured protein content using the bicinchoninic acid (BCA) method (Walker 1996) with a 'Compat-Able Protein Assay Preparation' kit (Pierce Biotechnology) and high molecular weight DOM (HMWDOM) as the amount of DOC retained in 12000 to $14000 \mathrm{Da}$ dialysis tubing after $24 \mathrm{~h}$ dialysis.

We tested for differences in chemical attributes among DOM sources using both univariate and multivariate statistics. For individual chemical attributes, we used univariate 1-way ANOVA with Tukey's HSD test. We adjusted the $\alpha$ value from 0.05 to 0.0028 , with a Bonferroni correction to account for multiple (18) comparisons. We also used principal component analysis (PCA) to describe the chemical characteristics of the DOM sources using 16 of the 18 chemical attributes (magnesium and manganese were excluded because of missing data). We used a correlation matrix of untransformed mean values in the PCA analysis because it standardizes data and thus does not overemphasize large values (Manly 1994).

Experimental design. We used batch cultures to quantify BP, BR, BGE and relative rates of carbon consumption on different sources and supplies of DOM. Each experimental unit consisted of a 11 polycarbonate bottle filled with $450 \mathrm{ml}$ of $0.22 \mu \mathrm{m}$-filtered lake water. We then inoculated each experimental unit with a $50 \mathrm{ml}$ aliquot of $2.7 \mu \mathrm{m}$-filtered (Whatman GF/D) lake water containing a natural microbial assemblage. We obtained lake water and the microbial community from
Norford Lake (Orange County, Vermont), which is an oligo-mesotrophic water body (chlorophyll $a=2.8$ to $\left.5.1 \mathrm{~g} \mathrm{~g} \mathrm{l}^{-1}\right)$ with relatively low DOC $(250 \mu \mathrm{M}$; Lennon 2004b). We manipulated DOM supply by exposing 11 experimental units to a concentration gradient ranging from 250 to $1400 \mu \mathrm{M}$ DOC. Using an unreplicated regression design, we then crossed the DOM supply manipulation with 6 different DOM sources (pine, hemlock, beech, maple, birch and oak). We incubated all 66 experimental units in environmental chambers at $20^{\circ} \mathrm{C}$ without light. We estimated BP $36 \mathrm{~h}$ after the initiation of the experiment by measuring the uptake and incorporation of ${ }^{3} \mathrm{H}$-leucine into bacterial protein (Kirchman 1993). We estimated BR as the change in dissolved oxygen concentrations between 24 and $48 \mathrm{~h}$ using spectrophotometric methods with corrections for color interference (Roland \& Cole 1999) created by each of the different DOM sources. We used a respiratory quotient of 1 to convert oxygen consumption to carbon evolution. We calculated BGE as (BP)/(BP + $\mathrm{BR})$. In addition to measuring the absolute rates of bacterial metabolism we also calculated the relative rates of carbon consumption as (BP + BR)/DOC.

We used multiple linear regression with indicator variables to evaluate the response of $\mathrm{BP}, \mathrm{BR}, \mathrm{BGE}$ and relative rates of carbon consumption to different sources and supplies of DOM. We used DOM concentration (measured as DOC) as a continuous predictor and DOM source as a qualitative predictor of bacterial metabolism (see indicator variables in Neter et al. 1996, p. 455-490). We examined residuals for assumptions of normality and homogeneity of variance, and used diagnostic tests (Studentized residuals, leverage, Cook's D, and Dffits) to identify potential outliers. We then calculated confidence limits (CL) around the differences between the slopes and intercepts for each possible pairing of DOM sources to determine whether DOM source affected bacterial metabolism along the supply gradient:

$$
\mathrm{CL}=\left(b_{i}-b_{j}\right) \pm\left(t_{\alpha[n-1]} \cdot \sqrt{\sigma_{b_{i}}^{2}+\sigma_{b_{j}}^{2}-2\left(\sigma_{b_{i} b_{j}}\right)}\right)
$$

where $b_{i}$ and $b_{j}$ are parameter estimates for 2 of the 6 DOM sources, $t$ is the critical value from the Student's $t$-distribution for a given $\alpha$ and sample size (n) used to create the parameters, $\sigma^{2}{ }_{b_{i}}$ and $\sigma^{2}{ }_{b_{j}}$ are the variances for each parameter estimate, and $\sigma_{b_{i} b_{j}}$ is the covariance between the 2 parameter estimates (Neter et al. 1996). We adjusted $\alpha$ with a Bonferroni correction to account for the 15 pairwise comparisons to determine whether different DOM sources affected the parameters of each metabolic response variable. We concluded that bacterial metabolism was not affected by DOM sources if adjusted confidence limits (99\%) around the difference of parameter estimates $\left(b_{i}-b_{j}\right)$ contained zero. 
Table 1. Chemical attributes of 6 different DOM sources used in bacterioplankton regrowth experiments. Values are mean $( \pm$ SE) of replicate samples from each DOM stock solution. Concentrations of all chemical attributes expressed as $\mu \mathrm{mol} \mathrm{mmol}^{-1} \mathrm{C}$ for each respective DOM source. We used univariate 1-way ANOVA with Tukey's HSD test to examine chemical differences between DOM sources and adjusted the $\alpha$ value from 0.05 to 0.0028 with a Bonferroni correction to account for the 18 multiple comparisons. Shared letters within a row indicate DOM sources with similar chemical concentrations. ${ }^{*}$ p-values that would be significant at an $\alpha$ of 0.05 , but were not considered significant after Bonferroni correction; -: concentrations outside range of our standard curve; HMWDOM: high molecular weight dissolved organic matter

\begin{tabular}{|c|c|c|c|c|c|c|c|c|c|}
\hline \multirow{2}{*}{$\begin{array}{l}\text { Element/ } \\
\text { Compound }\end{array}$} & \multirow[t]{2}{*}{ Beech } & \multirow[t]{2}{*}{ Oak } & \multirow[t]{2}{*}{ Hemlock } & \multirow[t]{2}{*}{ Birch } & \multirow[t]{2}{*}{ Maple } & \multirow[t]{2}{*}{ Pine } & \multicolumn{3}{|c|}{ ANOVA results } \\
\hline & & & & & & & df & $F$ & $\mathrm{p}$ \\
\hline Aluminum & $\begin{array}{c}3.8 \\
(0.08) \\
\mathrm{C}\end{array}$ & $\begin{array}{c}7.1 \\
(0.41) \\
b\end{array}$ & $\begin{array}{c}18.3 \\
(1.01) \\
\mathrm{a}\end{array}$ & $\begin{array}{c}6.9 \\
(0.31) \\
\mathrm{b}\end{array}$ & $\begin{array}{c}1.2 \\
(0.04) \\
d\end{array}$ & $\begin{array}{c}5.4 \\
(0.14) \\
\text { bc }\end{array}$ & 5,17 & 160.4 & $<0.0001$ \\
\hline Cadmium & $\begin{array}{c}4.3 \mathrm{E}-04 \\
(8.6 \mathrm{E}-05) \\
\mathrm{ab}\end{array}$ & $\begin{array}{c}3.2 \mathrm{E}-04 \\
(1.1 \mathrm{E} 0-4) \\
b\end{array}$ & $\begin{array}{c}4.3 \mathrm{E}-04 \\
(7.5 \mathrm{E}-05) \\
\mathrm{ab}\end{array}$ & $\begin{array}{c}5.4 \mathrm{E}-04 \\
(1.4 \mathrm{E}-04) \\
a b\end{array}$ & $\begin{array}{c}8.6 \mathrm{E}-04 \\
(7.5 \mathrm{E}-05) \\
\mathrm{a}\end{array}$ & $\begin{array}{c}4.3 \mathrm{E}-04 \\
(7.5 \mathrm{E}-05) \\
\mathrm{ab}\end{array}$ & 5,17 & 3.39 & $0.0386^{*}$ \\
\hline Calcium & $\begin{array}{c}9.4 \\
(0.47) \\
\mathrm{C}\end{array}$ & $\begin{array}{c}14.2 \\
(0.90) \\
\mathrm{b}\end{array}$ & $\begin{array}{c}9.7 \\
(0.83) \\
\text { C }\end{array}$ & $\begin{array}{c}7.5 \\
(0.68) \\
\mathrm{C}\end{array}$ & $\begin{array}{c}36.3 \\
(1.4) \\
\mathrm{a}\end{array}$ & $\begin{array}{c}6.5 \\
(0.62) \\
\mathrm{C}\end{array}$ & 5,26 & 152.2 & $<0.0001$ \\
\hline Chromium & $\begin{array}{c}7.0 \mathrm{E}-3 \\
(9.3 \mathrm{E}-4) \\
b\end{array}$ & $\begin{array}{c}7.0 \mathrm{E}-03 \\
(1.2 \mathrm{E}-03) \\
b\end{array}$ & $\begin{array}{c}1.8 \mathrm{E}-02 \\
(8.3 \mathrm{E}-04) \\
\mathrm{a}\end{array}$ & $\begin{array}{c}1.4 \mathrm{E}-02 \\
(1.9 \mathrm{E}-03) \\
\mathrm{a}\end{array}$ & $\begin{array}{c}9.3 \mathrm{E}-04 \\
(2.3 \mathrm{E}-02) \\
\mathrm{C}\end{array}$ & $\begin{array}{c}7.0 \mathrm{E}-03 \\
(2.3 \mathrm{E}-04) \\
b\end{array}$ & 5,17 & 46.0 & $<0.0001$ \\
\hline Copper & $\begin{array}{c}2.7 \mathrm{E}-02 \\
(7.6 \mathrm{E}-04) \\
\mathrm{ab}\end{array}$ & $\begin{array}{c}3.8 \mathrm{E}-02 \\
(1.3 \mathrm{E}-03) \\
\mathrm{a}\end{array}$ & $\begin{array}{c}3.0 \mathrm{E}-02 \\
(3.8 \mathrm{E}-04) \\
a b\end{array}$ & $\begin{array}{c}1.5 \mathrm{E}-02 \\
(5.7 \mathrm{E}-04) \\
\text { bc }\end{array}$ & $\begin{array}{c}3.6 \mathrm{E}-03 \\
(1.3 \mathrm{E}-03) \\
a\end{array}$ & $\begin{array}{c}4.2 \mathrm{E}-02 \\
(8.2 \mathrm{E}-03) \\
a\end{array}$ & 5,17 & 7.11 & 0.0026 \\
\hline HMWDOM & $\begin{array}{c}852 \\
(15.3) \\
a\end{array}$ & $\begin{array}{c}877 \\
(46.0) \\
\text { a }\end{array}$ & $\begin{array}{c}867 \\
(62.5) \\
\mathrm{a}\end{array}$ & $\begin{array}{c}683 \\
(8.2) \\
\mathrm{a}\end{array}$ & $\begin{array}{c}759 \\
(33.7) \\
a\end{array}$ & $\begin{array}{c}759 \\
(33.7) \\
a\end{array}$ & 5,16 & 3.23 & $0.049^{*}$ \\
\hline Humic acid & $\begin{array}{c}294 \\
(7.4) \\
\text { C }\end{array}$ & $\begin{array}{c}367 \\
(6.1) \\
a b\end{array}$ & $\begin{array}{c}399 \\
(2.8) \\
\text { a }\end{array}$ & $\begin{array}{c}384 \\
(7.3) \\
a b\end{array}$ & $\begin{array}{c}335 \\
(13.1) \\
\text { bc }\end{array}$ & $\begin{array}{c}369 \\
(19.1) \\
a b\end{array}$ & 5,17 & 12.6 & 0.0002 \\
\hline Iron & $\begin{array}{c}4.6 \\
(0.07) \\
C\end{array}$ & $\begin{array}{c}3.8 \\
(0.06) \\
d\end{array}$ & $\begin{array}{c}8.9 \\
(0.01) \\
\mathrm{a}\end{array}$ & $\begin{array}{c}8.1 \\
(0.02) \\
\mathrm{b}\end{array}$ & $\begin{array}{c}1.4 \\
(0.03) \\
\mathrm{f}\end{array}$ & $\begin{array}{c}3.4 \\
(0.04) \\
\mathrm{e}\end{array}$ & 5,19 & 2045 & $<0.0001$ \\
\hline Lead & $\begin{array}{c}1.5 \mathrm{E}-02 \\
(7.0 \mathrm{E}-04) \\
b\end{array}$ & $\begin{array}{c}3.5 \mathrm{E}-03 \\
(1.2 \mathrm{E}-04) \\
\mathrm{d}\end{array}$ & $\begin{array}{c}2.0 \mathrm{E}-02 \\
(2.9 \mathrm{E}-04) \\
\mathrm{a}\end{array}$ & $\begin{array}{c}1.9 \mathrm{E}-02 \\
(9.9 \mathrm{E}-04) \\
\text { a }\end{array}$ & $\begin{array}{c}4.1 \mathrm{E}-03 \\
(7.0 \mathrm{E}-04) \\
d\end{array}$ & $\begin{array}{c}1.1 \mathrm{E}-02 \\
(6.4 \mathrm{E}-02) \\
\mathrm{C}\end{array}$ & 5,17 & 127.4 & $<0.0001$ \\
\hline Magnesium & $\begin{array}{c}0.19 \\
(0.006) \\
d\end{array}$ & $\begin{array}{c}1.29 \\
(0.069) \\
b\end{array}$ & - & - & $\begin{array}{c}3.37 \\
(0.079) \\
\text { a }\end{array}$ & $\begin{array}{c}1.04 \\
(0.030) \\
\mathrm{C}\end{array}$ & 3,11 & 594.5 & $<0.0001$ \\
\hline Manganese & $\begin{array}{c}0.68 \\
(0.02) \\
\mathrm{b}\end{array}$ & $\begin{array}{c}0.90 \\
(0.026) \\
\text { a }\end{array}$ & $\begin{array}{c}0.53 \\
(0.009) \\
\text { C }\end{array}$ & $\begin{array}{c}0.86 \\
(0.022) \\
\text { a }\end{array}$ & - & $\begin{array}{c}0.44 \\
(0.020) \\
\text { C }\end{array}$ & 4,14 & 95.2 & $<0.0001$ \\
\hline Total N & $\begin{array}{c}79.1 \\
(1.09) \\
\mathrm{a}\end{array}$ & $\begin{array}{c}92.4 \\
(4.90) \\
\mathrm{a}\end{array}$ & $\begin{array}{c}70.3 \\
(2.88) \\
\mathrm{b}\end{array}$ & $\begin{array}{c}77.7 \\
(2.63) \\
\mathrm{a}\end{array}$ & $\begin{array}{c}83.0 \\
(4.52) \\
\mathrm{a}\end{array}$ & $\begin{array}{c}79.6 \\
(1.37) \\
\mathrm{a}\end{array}$ & 5,23 & 6.88 & $0.0112^{*}$ \\
\hline Total P & $\begin{array}{c}7.3 \\
(0.11) \\
\mathrm{a}\end{array}$ & $\begin{array}{c}7.1 \\
(0.12) \\
a b\end{array}$ & $\begin{array}{c}6.7 \\
(0.13) \\
b\end{array}$ & $\begin{array}{c}5.7 \\
(0.17) \\
\mathrm{C}\end{array}$ & $\begin{array}{c}3.7 \\
(0.11) \\
\mathrm{e}\end{array}$ & $\begin{array}{c}4.2 \\
(0.09) \\
d\end{array}$ & 5,17 & 276.9 & $<0.0001$ \\
\hline Polyphenol & $\begin{array}{c}2.6 \\
(0.03) \\
\text { ab }\end{array}$ & $\begin{array}{c}2.2 \\
(0.02) \\
d\end{array}$ & $\begin{array}{c}2.6 \\
(0.07) \\
\text { ab }\end{array}$ & $\begin{array}{c}2.7 \\
(0.02) \\
\mathrm{a}\end{array}$ & $\begin{array}{c}2.5 \\
(0.03) \\
\text { bc }\end{array}$ & $\begin{array}{c}2.5 \\
(0.09) \\
\text { bc }\end{array}$ & 5,17 & 29.3 & $<0.0001$ \\
\hline Potassium & $\begin{array}{c}3.2 \\
(0.09) \\
\mathrm{b}\end{array}$ & $\begin{array}{c}4.0 \\
(0.01) \\
\mathrm{a}\end{array}$ & $\begin{array}{c}2.5 \\
(0.03) \\
\mathrm{C}\end{array}$ & $\begin{array}{c}2.0 \\
(0.07) \\
d\end{array}$ & $\begin{array}{c}2.7 \\
(0.04) \\
\text { C }\end{array}$ & $\begin{array}{c}2.7 \\
(0.04) \\
\text { C }\end{array}$ & 5,17 & 162.2 & $<0.0001$ \\
\hline Protein & $\begin{array}{c}3.2 \\
(0.12) \\
d\end{array}$ & $\begin{array}{c}4.6 \\
(0.14) \\
\mathrm{a}\end{array}$ & $\begin{array}{c}3.4 \\
(0.08) \\
\mathrm{cd}\end{array}$ & $\begin{array}{c}3.5 \\
(0.05) \\
\text { bcd }\end{array}$ & $\begin{array}{c}3.8 \\
(0.07) \\
\text { bc }\end{array}$ & $\begin{array}{c}3.9 \\
(0.13) \\
\mathrm{b}\end{array}$ & 5,17 & 24.4 & $<0.0001$ \\
\hline Sodium & $\begin{array}{c}169 \\
(1.9) \\
\mathrm{C}\end{array}$ & $\begin{array}{c}183 \\
(2.1) \\
\mathrm{b}\end{array}$ & $\begin{array}{c}233 \\
(3.1) \\
\mathrm{a}\end{array}$ & $\begin{array}{c}98 \\
(1.3) \\
\mathrm{f}\end{array}$ & $\begin{array}{c}107 \\
(1.3) \\
\mathrm{e}\end{array}$ & $\begin{array}{c}127 \\
(1.5) \\
d\end{array}$ & 5,25 & 827.1 & $<0.0001$ \\
\hline Zinc & $\begin{array}{c}0.07 \\
(0.001) \\
b\end{array}$ & $\begin{array}{c}0.04 \\
(0.002) \\
d\end{array}$ & $\begin{array}{c}0.04 \\
(0.001) \\
d\end{array}$ & $\begin{array}{c}0.06 \\
(0.001) \\
\text { C }\end{array}$ & $\begin{array}{c}0.06 \\
(0.001) \\
\text { C }\end{array}$ & $\begin{array}{c}0.08 \\
(0.001) \\
\text { a }\end{array}$ & 5,17 & 135.3 & $<0.0001$ \\
\hline
\end{tabular}


Table 2. Weightings for the first 3 principal components (PC) generated from a matrix of 16 chemical attributes for 6 different DOM sources. Chemistry data represent untransformed concentrations scaled between leachates to $\mu \mathrm{mol} \mathrm{mmol}^{-1} \mathrm{C}$. HMWDOM: high molecular weight dissolved organic matter

\begin{tabular}{|lccr|}
\hline Chemical attributes & PC 1 & PC 2 & PC 3 \\
\hline Total nitrogen & -0.303 & 0.095 & 0.210 \\
Copper & -0.265 & 0.163 & 0.054 \\
Protein & -0.259 & 0.200 & 0.338 \\
Calcium & -0.247 & -0.137 & 0.068 \\
Potassium & -0.218 & 0.351 & -0.177 \\
Cadmium & -0.102 & -0.356 & 0.133 \\
Zinc & -0.044 & -0.311 & -0.271 \\
HMWDOM & -0.024 & 0.420 & -0.223 \\
Sodium & 0.143 & 0.400 & -0.088 \\
Humic acids & 0.161 & 0.085 & 0.572 \\
Total phosphorus & 0.179 & 0.221 & -0.448 \\
Aluminum & 0.287 & 0.244 & 0.209 \\
Polyphenolics & 0.290 & -0.297 & -0.109 \\
Chromium & 0.352 & 0.095 & 0.229 \\
Iron & 0.369 & 0.069 & 0.128 \\
Lead & 0.381 & -0.070 & -0.042 \\
\% variance explained & 42 & 29 & 15 \\
\hline
\end{tabular}

We defined the slopes of $\mathrm{BP}$ and $\mathrm{BR}$ versus DOC as rates of carbon-specific metabolism, then attempted to explain variance in carbon-specific metabolism as a function of DOM chemistry. First, we used the output from the PCA of the DOM chemistry to explain carbonspecific metabolism. We used eigenvectors from the first 3 principal component (PC) axes as individual predictors of the BP-DOC and BR-DOC slopes. Second, we used individual chemical attributes as predictors of carbon-specific metabolism. We narrowed down the number of individual predictors by constructing a correlation matrix comparing all of the chemical attributes to the BP-DOC and BR-DOC slopes. We considered all individual chemical variables that had high correlation coefficients $(>|0.7|$, see Fowler et al. 1998) with $\mathrm{BP}-\mathrm{DOC}$ and BR-DOC slopes as potential predictors of carbonspecific metabolism. We used curve-fitting (SigmaPlot Version 8.0, 2002) to describe the relationships between carbon-specific metabolism and the selected group of chemical predictor variables. We used SAS (SAS 1999) for all other statistical procedures.

\section{RESULTS}

\section{Chemical composition of DOM}

The DOM sources were chemically different from one another. All the individual chemical attributes were different at $\alpha=0.05$ (1-way ANOVA), but cad- mium, HMWDOM, and TN were not significantly different after applying a Bonferroni correction for multiple comparisons (Table 1). PCA also indicated that the 6 DOM sources had different chemical compositions. The first 3 PC axes explained $86 \%$ of the chemical variation in the different DOM sources (Table 2, Fig. 1). Hemlock DOM was positively correlated with PCA Axis 1 (iron and lead), while maple DOM was negatively correlated with PCA Axis 1 (TN). Oak DOM was positively correlated with PCA Axis 2 (HMWDOM and sodium), while birch and maple DOM were negatively correlated with PCA Axis 2 (cadmium and zinc). Finally, beech DOM was negatively correlated with PCA Axis 3 (TP).

\section{Bacterial productivity}

Bacterial productivity was affected by both DOC concentration and the different DOM sources. The
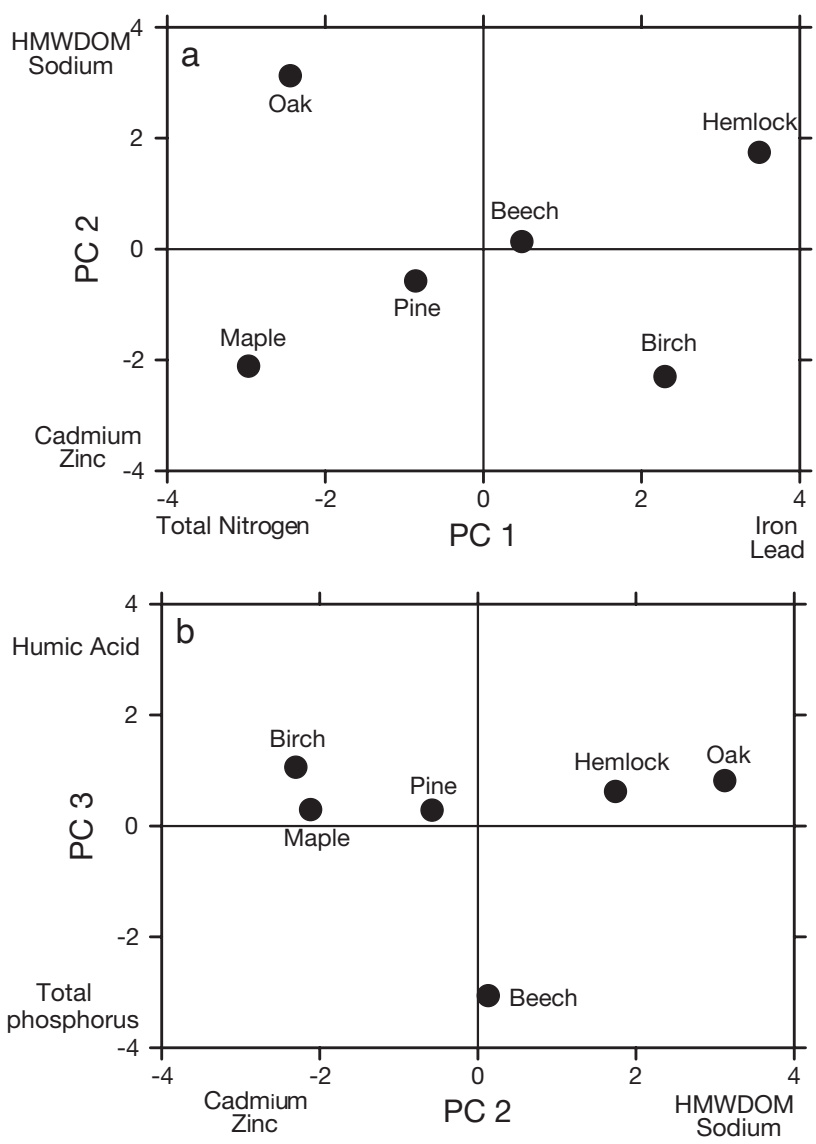

Fig. 1. Principal component analysis (PCA) of chemical attributes of 6 terrestrial DOM sources used in the bacterioplankton batch culture experiments. Data points shown for first 3 principal component axes. (a) PC2 versus PC1, (b) PC3 versus PC2. HMWDOM: high molecular weight dissolved organic matter 
Table 3. Parameter estimates for bacterial productivity and bacterial respiration (mean $\pm \mathrm{SE}$ ) along a DOC concentration gradi-

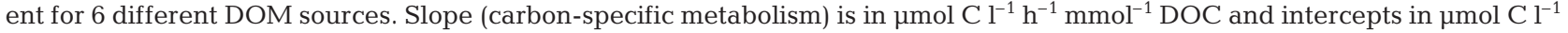
$\mathrm{h}^{-1}$. Shared letters within a column identify parameters not statistically different from each other as determined by pairwise comparisons with $99 \%$ confidence limits

\begin{tabular}{|c|c|c|c|c|c|c|c|c|c|c|c|c|}
\hline \multirow{3}{*}{$\begin{array}{l}\text { DOM source } \\
\text { Beech }\end{array}$} & \multicolumn{6}{|c|}{ Bacterial productivity } & \multicolumn{6}{|c|}{ Bacterial respiration } \\
\hline & \multicolumn{3}{|c|}{ Intercept } & \multicolumn{3}{|c|}{ Slope } & \multicolumn{3}{|c|}{ Intercept } & \multicolumn{3}{|c|}{ Slope } \\
\hline & 0.17 & $(0.136)$ & $\mathrm{ab}$ & 1.8 & $(0.24)$ & $\mathrm{a}$ & -0.29 & $(0.358)$ & $\mathrm{a}$ & 2.2 & $(0.55)$ & a \\
\hline Oak & -0.01 & $(0.121)$ & $\mathrm{a}$ & 1.6 & $(0.20)$ & $\mathrm{ab}$ & -0.23 & $(0.366)$ & $\mathrm{a}$ & 1.8 & $(0.60)$ & $\mathrm{a}$ \\
\hline Hemlock & 0.49 & $(0.120)$ & $\mathrm{b}$ & 1.1 & $(0.19)$ & $\mathrm{abc}$ & 0.16 & $(0.363)$ & $\mathrm{a}$ & 1.9 & $(0.59)$ & $\mathrm{a}$ \\
\hline Birch & 0.30 & $(0.082)$ & $a b$ & 0.8 & $(0.12)$ & bc & -0.20 & $(0.247)$ & $\mathrm{a}$ & 1.5 & $(0.36)$ & $\mathrm{a}$ \\
\hline Maple & 0.27 & $(0.127)$ & $a b$ & 0.6 & $(0.22)$ & $\mathrm{C}$ & 0.33 & $(0.384)$ & $\mathrm{a}$ & 1 & $(0.67)$ & $\mathrm{a}$ \\
\hline Pine & 0.42 & $(0.082)$ & $\mathrm{b}$ & 0.6 & $(0.17)$ & $\mathrm{C}$ & -0.12 & $(0.354)$ & $\mathrm{a}$ & 1.8 & $(0.53)$ & $\mathrm{a}$ \\
\hline
\end{tabular}

indicator variables multiple regression model explained $88 \%$ of the variation in $\operatorname{BP}\left(F_{11,71}=37.8, \mathrm{p}<\right.$ 0.0001). Carbon-specific productivity was approximately $3 \times$ higher on beech and oak DOM sources than on maple and pine DOM sources (Table 3). Carbonspecific productivity was intermediate on hemlock and birch DOM sources and could not be statistically distinguished from carbon-specific productivity on the other DOM sources (Table 3).

We removed one BP data point from the multiple regression model. $\mathrm{BP}$ in the beech treatment measured $1.5 \mu \mathrm{mol} \mathrm{l} \mathrm{l}^{-1} \mathrm{~h}^{-1}$ at $1250 \mu \mathrm{m}$ DOC, creating a humpedshape relationship (see Fig. 2). Diagnostic checks from the multiple regression (Studentized residuals, leverage, Cook's $D$, and Dffits) indicated that this data point weighed heavily in the analysis. When this point was included, however, we could fit the data well for this DOM source by including a polynomial term in a regression analysis:

$$
\begin{gathered}
\mathrm{BP}_{(\text {beech })}=-0.4+0.005(\mathrm{DOC})-0.000005(\mathrm{DOC})^{2} \\
\mathrm{R}^{2}=0.93, \mathrm{p}<0.0001
\end{gathered}
$$

Eigenvectors from the PCA were poor predictors of carbon-specific productivity ( $p>0.05$ ). Instead, our correlation analyses identified 3 chemical attributes as potential predictors of carbon-specific productivity: TP $(\mathrm{r}=0.90)$, HMWDOM $(\mathrm{r}=0.73)$, and potassium $(\mathrm{r}=$ 0.72). We did not identify models where HMWDOM or potassium was a significant predictor of carbon-specific productivity ( $p>0.05$ ), but carbon-specific productivity was explained by phosphorus content. We represent this relationship as an exponential function of the carbon:phosphorus ratio (C:P) of the DOM sources (see Fig. 3):

$$
\begin{gathered}
\text { Carbon-specific productivity }=0.05+329 \mathrm{e}^{(-0.05 \cdot \mathrm{C}: \mathrm{P})} \\
\qquad \mathrm{R}^{2}=0.99, \mathrm{p}<0.0003
\end{gathered}
$$

Phosphorus content was not correlated with any of the other chemical attributes measured on the 6 DOM sources $(p>0.05)$.

\section{Bacterial respiration}

BR increased significantly over the DOC concentration gradient, but was not affected by DOM source (Fig. 2, Table 3). Multiple regression using DOC concentration and DOM source as predictors explained $67 \%$ of the variation in $\operatorname{BR}\left(F_{11,70}=10.9, \mathrm{p}<0.0001\right)$. We did not attempt to use DOM chemical attributes to explain variance in carbon-specific respiration because BR was not significantly affected by the different DOM sources (Table 3).

\section{Bacterial growth efficiency}

BP and BR were significantly correlated with one another in all treatments except maple DOM (Table 4). As such, BGE was not affected by DOM concentration or DOM source $\left(\mathrm{R}^{2}=0.23, F_{11,63}=1.4, \mathrm{p}=0.20\right)$. BGE averaged $0.54 \pm 0.022(\mathrm{SE})$ for all samples.

\section{Relative rates of carbon consumption}

The relative rates of carbon consumption were not affected by the concentration of DOC, but were apparently influenced by the source of DOM $\left(R^{2}=0.39\right.$;

Table 4. Correlation between bacterial respiration (BR) and bacterial productivity (BP) for 6 different sources of terrestrial-derived DOM

\begin{tabular}{|lcc|}
\hline DOM source & Correlation coefficient $(\mathrm{r})$ & $\mathrm{p}$-value \\
\hline Pine & 0.62 & 0.0404 \\
Birch & 0.80 & 0.0032 \\
Hemlock & 0.68 & 0.0203 \\
Beech & 0.90 & 0.0004 \\
Oak & 0.78 & 0.0045 \\
Maple & 0.07 & 0.8473 \\
\hline
\end{tabular}


$\left.F_{11,64}=3.05, \mathrm{p}<0.003\right)$. Relative rates of carbon consumption were significantly higher on hemlock DOM when compared to the rates of the other pooled DOM sources $(p=0.004)$. However, pairwise comparisons revealed that there were no significant differences in the relative rates of carbon consumption among the different DOM sources. Relative rates of carbon consumption ranged from 1 to $18 \% \mathrm{~d}^{-1}$; the average rate for all samples was $7 \pm 0.3 \% \mathrm{~d}^{-1}$ (SEM).

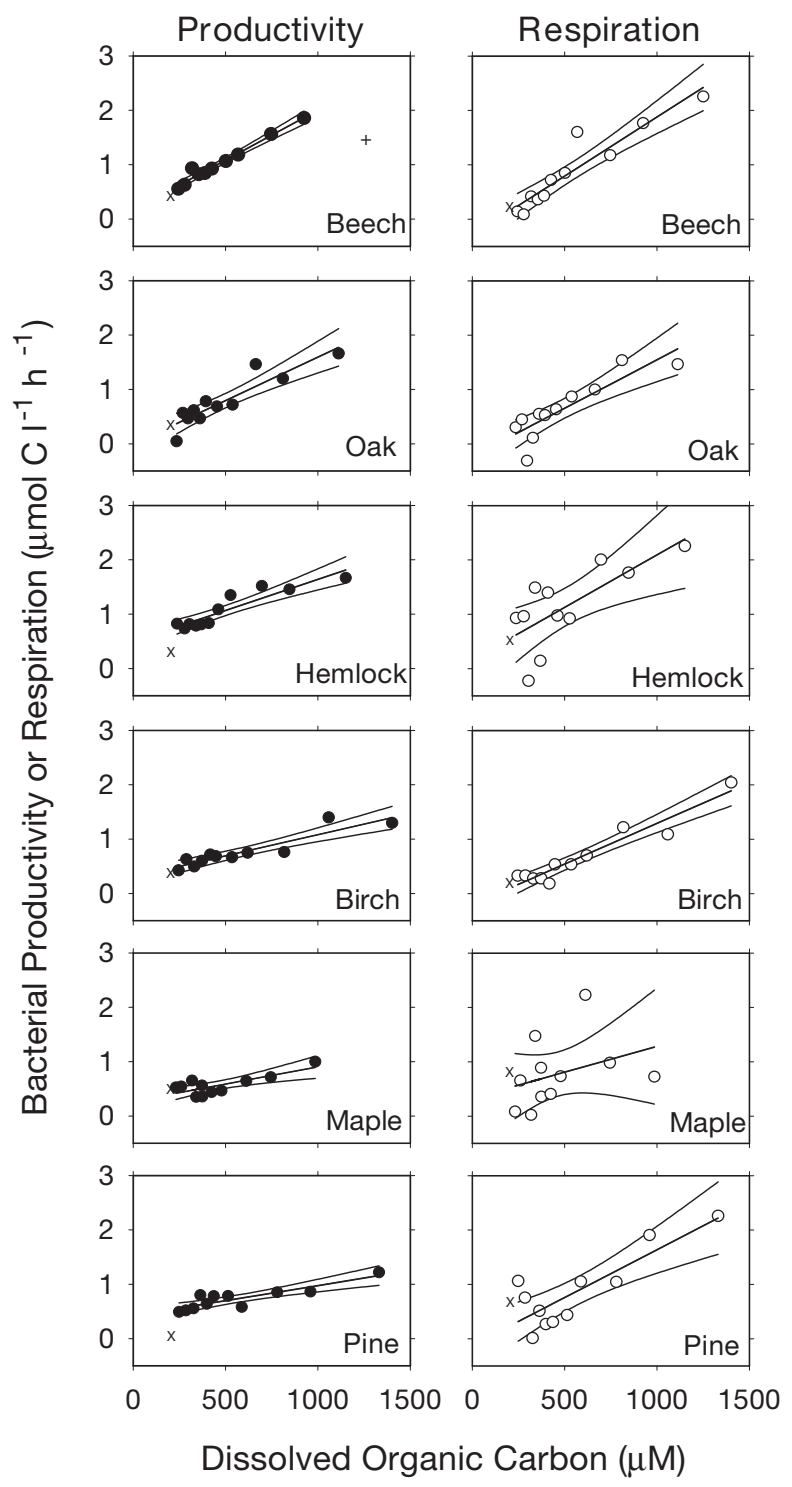

Fig. 2. Bacterial productivity and bacterial respiration in response to different concentrations and sources of terrestrialderived DOM. Graphs presented in descending order of carbonspecific bacterial productivity (i.e. the slopes). Lines: predicted values and $95 \%$ confidence intervals from multiple regression models $s_{i}+$ : productivity outlier detected and removed from multiple regression analysis, but included in a separate polynomial regression (see second subsection of 'Results'); x: bacterial productivity and bacterial respiration measurements in ambient lake water not included in multiple regression analyses

\section{DISCUSSION}

Terrestrial-derived DOM can serve as carbon and nutrient subsidies for bacterioplankton in both freshwater and marine ecosystems. The consumption of this allochthonous resource should influence bacterial metabolism and may in turn have consequences for food webs and the biogeochemistry of aquatic ecosystems. This study examined how variation in the source and supply of DOM affects the metabolism of lake bacterioplankton. We estimated that bacteria consumed approximately $7 \%$ of the bulk DOC pool, which is less than the consumption reported for some lakes (14\%), rivers $(19 \%)$, and marine environments (19\%) (Søndergaard \& Middelboe 1995). Nevertheless, both BP and BR increased linearly with increasing supply of terrestrial DOM. In addition, BP was strongly affected by the DOM source. In particular, DOP content was a good predictor of DOM quality for BP.

\section{Bacterial metabolism and DOM supply}

We predicted that BP and BR would generally increase with additions of DOM because heterotrophic bacterioplankton are often carbon-limited (Kirchman \& Rich 1997). However, we did not assume that metabolic rates would be constant along the DOM gradient. For example, radiotracer studies (i.e. ${ }^{14} \mathrm{C}$-labeled glucose, leucine, acetate) commonly reveal that that carbon uptake rates of microbial communities saturate at high substrate concentrations according to MichaelisMenten dynamics (Wright \& Hobbie 1965, Garnier \& Benest 1991, Münster 1993). Such kinetic constraints could affect resource uptake and subsequent biomass production. In addition, the functional relationship between metabolism and DOM could be shaped by the relative concentrations of co-limiting nutrients (e.g. nitrogen, phosphorus, etc.). However, we found little evidence that bacterial metabolism saturated with increasing DOM supply. Instead, both BP and BR increased linearly along the experimental supply gradient (Table 3, Fig. 2).

Only in the beech treatment did it appear that metabolic rates might have changed along the DOM supply gradient (Fig. 2). At the highest DOC concentration, BP in the beech treatment was $63 \%$ lower than predicted based on the linear equation in Table 3 . The inclusion of the outlier data point resulted in a hump-shaped relationship so that BP actually decreased at the highest DOC concentration instead of saturating at a maximum level of productivity. In contrast, BR remained linear along the DOC gradient, suggesting that BP and $\mathrm{BR}$ were decoupled at high levels of metabolism in this treatment. 
The positive relationship between bacterial metabolism and DOM supply in our study is consistent with some trends found in freshwater and marine ecosystems. For example, both BP and BR of coastal microbial communities increased near linearly when exposed to 3 levels of high molecular weight carbon ( $>1 \mathrm{kDa}$ ) ranging from 116 to $302 \mu \mathrm{M}$ DOC (Amon \& Benner 1996). Similarly, BR in the Bay of Biscay increased over a relatively narrow DOC gradient ( 72 to $109 \mu \mathrm{M}$ ) that was associated with a coastal upwelling event (Gonzalez et al. 2003). In lake communities, bacterial biomass production increased with experimental additions of aged humic material (Eiler et al. 2003). Finally, crosssystem analyses of batch culture experiments have shown that the proportion of labile DOC (Søndergaard \& Middelboe 1995) and the rate of DOC consumption (del Giorgio \& Davis 2003) increase with increasing bulk DOC concentration.

However, an equally large number of studies have failed to detect patterns between bacterioplankton metabolism and bulk DOM concentration. For example, there was no relationship between either bacterial growth or BP along a DOC gradient (208 to $758 \mu \mathrm{M}$ ) in 20 Quebec lakes (Smith \& Prairie 2004). Likewise, BP was unaffected by DOC concentrations in tropical coastal lagoons (Farjalla et al. 2002). In tidal freshwater stretches of the Hudson River, 10 years of data have revealed that there is no relationship between $\mathrm{BP}$ and DOC (125 to $583 \mu \mathrm{M}$ ) (Findlay 2003). The null relationships found in this latter group of studies suggest that factors besides bulk DOM content, such as DOM chemistry, may be important in driving the metabolism of heterotrophic bacterioplankton.

Contrary to our initial predictions, BGE did not increase over our DOM concentration gradient. Under low DOM supplies, we anticipated that most assimilated carbon would be devoted to maintenance costs, resulting in low BGE (Russell \& Cook 1995). Occasionally, BGE does not increase with substrate concentration because resources are not always sufficiently energy-rich for bacteria to reduce all of the available organic matter into cellular carbon (del Giorgio \& Cole 1998). However, this explanation cannot account for the lack of pattern between BGE and DOM since BP always responded positively to increased DOM concentrations (Fig. 2, Table 3). Instead, the lack of a BGE response in this study most probably reflects our experimental approach. Bacterial growth is often unconstrained on a given resource in batch cultures that are conducted over relatively short time periods. Under such conditions, BP and BR will often be correlated (e.g. Table 4), leading to a relatively constant BGE. Additionally, a lack of a relationship between DOC and BGE may have arisen if bacterial growth rates were decoupled from ${ }^{3} \mathrm{H}$-leucine incorporation rates. In general, our results are consistent with the empirical trend that BGE plateaus at approximately 0.5

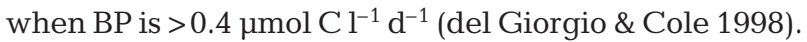

\section{Chemical attributes of different DOM sources}

Our leaching approach created DOM sources with different chemical characteristics (Tables 1 \& 2, Fig. 1). It is important to note, however, that it was not our explicit objective to test hypotheses about how the identity of terrestrial vegetation influences DOM chemistry. Nevertheless, attributes of our DOM sources are consistent with some vegetation-soil chemistry patterns reported in the literature. For example, our hemlock leachate had high concentrations of aluminum, iron and lead (Table 1, Fig. 1), consistent with soils in coniferous forests (David \& Driscoll 1984, LaZerte \& Scott 1996) and probably reflecting the fact that metals are mobilized by the production of organic acids in hemlock stands (Dijkstra et al. 2001). Conifers also have low foliar nitrogen relative to most deciduous species and consequently produce litter with high C:N ratios (Templer et al. 2003). This pattern was reflected in our hemlock leachate (lowest nitrogen concentration), but not in the pine leachate (intermediate nitrogen concentration). Finally, our maple DOM source had relatively high concentrations of nitrogen, calcium and magnesium, but low concentrations of aluminum, consistent with the chemical attributes commonly associated with soils in sugar maple stands (Minocha et al. 2000, Dijkstra \& Smits 2002, Lovett \& Mitchell 2004).

Despite similarities between our DOM chemistry and the soil chemistry patterns in the literature, we must be cautious about using our data to link specific forest compositions to the metabolism of terrestrial DOM by aquatic microorganisms. First, there were most likely some biases in our extraction process, although the percentages of HMWDOM and polyphenolic compounds are generally consistent with other reports (e.g. Thurman 1985, Engelhaupt \& Bianchi 2001). Second, our approach does not address whether the chemical signal of different DOM sources would be preserved along land-water flowpaths of DOM (see Sommer et al. 1997). For example, soil microorganisms may modify chemical attributes of DOM (Smolander \& Kitunen 2002, Marschner \& Kalbitz 2003) before it reaches aquatic ecosystems.

\section{Bacterial metabolism on different DOM sources}

Variation in the chemical composition of DOM can modify aquatic microbial metabolism (e.g. Vallino et al. 1996, Sun et al. 1997). Despite variation in a suite of 
chemical attributes, our results suggest that the phosphorus content of DOM is an important determinant of BP. We observed a $3 \times$ exponential decline in carbonspecific productivity with decreasing phosphorus content when expressed as a C:P ratio (Fig. 3). Since we did not manipulate phosphorus directly, it is possible that this trend represents a correlation with other unmeasured attributes of our DOM sources. However, multiple lines of evidence suggest that phosphorus content should be an important factor influencing DOM quality for aquatic bacteria.

Phosphorus often limits or co-limits bacterial metabolism in freshwater (Currie \& Kalff 1984, Pace \& Cole 1996, Drakare 2002) and marine ecosystems (Rivkin \& Anderson 1997, Sala et al. 2002). The high phosphorus demand of aquatic bacteria is reflected by their relatively low biomass C:P ratios (8 to $173: 1$ by moles; Chrzanowski et al. 1996, Hochstadter 2000, Vrede et al. 2002). In contrast, water-column ratios of DOC:DOP are typically much higher (400 to $800: 1$ by mol; Hopkinson et al. 1997, McKnight et al. 1997). The C:P ratios of our DOM sources were slightly lower (137 to 275:1, by mol), but were still higher than Redfield values (106:1 by mol).

As a result, carbon-specific productivity was relatively high on DOM sources with low C:P ratios. In contrast, carbon-specific productivity dropped when substrate C:P ratios moved outside the reported stoichiometric range required for optimum bacterial biomass production (8 to $173: 1$ by mol; Fig. 3). Together, this information suggests that phosphorus demands of rapidly growing bacteria may be alleviated by the natural variation found among different sources of terrestrial DOM. In addition, higher DOP may allow bacteria to consume more recalcitrant carbon molecules (Benner et al. 1988), possibly through increased extracellular enzyme production (Sinsabaugh et al. 1997).

In contrast with $\mathrm{BP}$, we found no effect of different DOM sources on carbon-specific respiration. There are 2 potential explanations for this lack of pattern. First, it is possible that BR might not be affected by different DOM sources because of a slight decoupling of catabolic and anabolic respiratory reactions (del Giorgio \& Cole 1998). BP and BR were not perfectly correlated (Table 4), and thus anabolic respiratory costs could be masked by the independent effect of different DOM sources on catabolic respiratory costs. Second, we may not have been able to detect an underlying effect of different DOM sources because of the inherent variability associated with BR measurements (see del Giorgio \& Cole 1998). For example, the coefficient of variation $(\mathrm{CV})$ for replicate $\mathrm{BP}$ measurements on an experimental unit was only $3 \%$, whereas the $\mathrm{CV}$ for replicate BR measurements on an experimental unit was $35 \%$ (data not shown). This variability should not

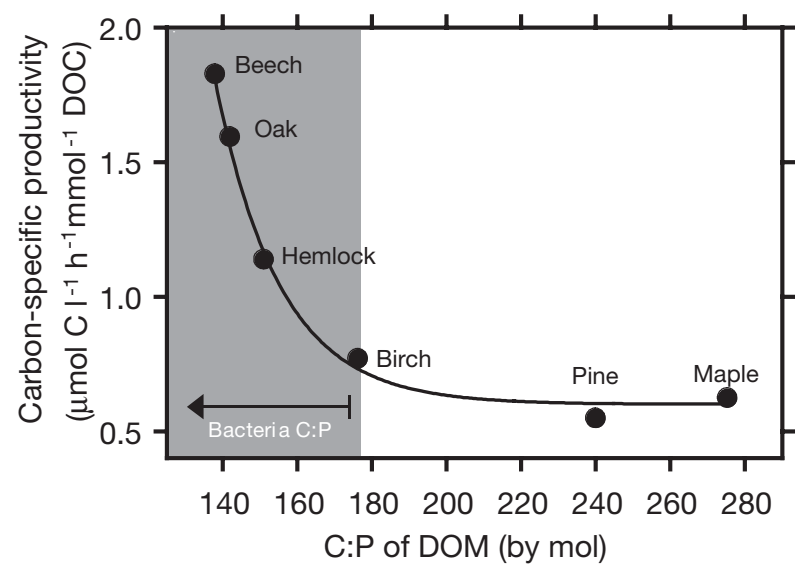

Fig. 3. Carbon-specific bacterial productivity (slopes of the bacterial productivity versus DOC relationship in Fig. 2 \& Table 3) declined exponentially with C:P ratio of different DOM sources. Shaded area: range of C:P ratio of aquatic bacterial biomass reported in the literature (Chrzanowski et al. 1996, Hochstadter 2000, Vrede et al. 2002)

influence our parameter estimates (Miller 1986), but would increase the confidence interval around the parameter estimates and thus reduce our ability to detect significant effects of different DOM sources on BR. Obviously this variability in BR also affects our estimates of BGE.

In contrast with a number of studies, BGE was not affected by DOM source with varying chemical attributes. For example, BGE can be influenced by DOM characteristics such as mineral nutrient content (Benner et al. 1988), molecular weight (Tranvik 1990), amino acid content (Carlson \& Ducklow 1996) and carbon:nutrient ratios (Goldman \& Dennett 2000). However, these patterns are not always consistent between studies (del Giorgio \& Cole 1998).

Often, BGE is correlated with factors that drive BP. For example, BP was more sensitive to environmental conditions than BR in the Hudson River, and thus BP was largely responsible for the observed changes in BGE (Roland \& Cole 1999). However, in the present study, the magnitudes of response for BP and BR to DOM manipulations were roughly equal (Table 4). As such, BGE remained relatively constant, despite substantial variation in key chemical attributes of DOM that sometimes affect bacterial metabolism.

In theory, if BP changed with DOM quality and BR remained constant (e.g. Fig. 2, Table 3), then one would predict that BGE must have also changed with DOM quality. We were unable to detect this pattern with our data. As mentioned above, the variability of our BR measurements may have prevented us from detecting an underlying effect of DOM quality on BGE. However, the predicted values generated from our multiple regression analyses represent unbiased esti- 
mates of BP and BR. A slightly different pattern emerged when we estimated BGE from predicted instead of observed BP and BR. Predicted BGE ranged from 0.25 to 0.47 and qualitatively declined with increasing C:P of the DOM sources, although this relationship was not statistically significant $(\mathrm{p}=0.19, \mathrm{n}=$ 6). An analysis with more power may have revealed that there was a true relationship between BGE and the phosphorus content of DOM.

\section{CONCLUSION}

Our results contribute to a growing body of literature which documents the significance of terrestrial DOM as a carbon subsidy for microorganisms in aquatic ecosystems. More importantly, our findings indicate that inputs of certain DOM sources alleviate phosphorus-limited bacterial biomass production. DOM sources from beech, oak and hemlock soils had elevated concentrations of phosphorus that resulted in relatively high rates of carbon-specific productivity. These results suggest there may be strong links between the terrestrial vegetation, soil chemistry, and aquatic microbial metabolism. However, there is currently a lack of data linking vegetation type and phosphorus content of soil DOM (Chantigny 2003). In addition, more detailed studies are needed to explore the dynamics of DOM chemistry along watershed flowpaths. For example, it is well known that soils have a high phosphorus sorption capacity (Frossard et al. 1995) relative to other nutrients and that soil microorganisms modify DOM chemistry prior to being exported to nearby waterbodies (Marschner \& Kalbitz 2003). Comparative surveys of aquatic bacterial metabolism in watersheds with contrasting forest composition would be one useful approach to test the results of this laboratory study.

Acknowledgements. We acknowledge K. L. Cottingham, S. Kaushal, M.L. Pace and 2 anonymous referees for critical review of the manuscript. We thank M.L. Pace for use of BOD bottles, J. J. Cole for advice on measuring respiration rates, S. Findlay for insightful discussion, the Norford Lake Association for lake access and boating equipment, A.J. Friedland and P. Zietz for ICP-AES analysis, X. Feng and C. E. Renshaw for use of their TIC/TOC analyzer, and J.M. Palange for field assistance. This research was supported by NSF-DDIG 0206531 (J.T.L. and K. L. Cottingham), USGS/ NIWR 2002NH1B (J.T.L. and K. L. Cottingham), and a Dartmouth Graduate Alumni Research Award (J.T.L.).

\section{LITERATURE CITED}

Aitkenhead-Peterson JA, McDowell WH, Neff JC (2003) Sources, production, and regulation of allochthonous dissolved organic matter inputs to surface waters. In: Findlay SEG, Sinsabaugh RL (eds) Aquatic ecosystems: inter- activity of dissolved organic matter. Academic Press, New York, p 26-70

Amon RMW, Benner R (1996) Bacterial utilization of different size classes of dissolved organic matter. Limnol Oceanogr 41:41-51

APHA (American Public Health Association) (1998) Standard methods for the examination of water and wastewater. APHA, Washington, DC

Bano N, Moran MA, Hodson RE (1997) Bacterial utilization of dissolved humic substances from a freshwater swamp. Aquat Microb Ecol 12:233-238

Benner R, Lay J, Knees E, Hodson RE (1988) Carbon conversion efficiency for bacterial growth on lignocelluloseimplications for detritus-based food webs. Limnol Oceanogr 33:1514-1526

Bertilsson S, Jones JB (2003) Supply of dissolved organic matter to aquatic ecosystems: autochthonous sources. In: Findlay SEG, Sinsabaugh RL (eds) Aquatic ecosystems: interactivity of dissolved organic matter. Academic Press, New York, p 3-25

Biddanda B, Ogdahl M, Cotner J (2001) Dominance of bacterial metabolism in oligotrophic relative to eutrophic waters. Limnol Oceanogr 46:730-739

Boyer EW, Hornberger GM, Bencala KE, McKnight DM (2000) Effects of asynchronous snowmelt on flushing of dissolved organic carbon: a mixing model approach. Hydrol Process 14:3291-3308

Canham CD, Pace ML, Papaik MJ, Primack AGB, Roy KM, Maranger RJ, Curran RP, Spada DM (2004) A spatiallyexplicit watershed-scale analysis of dissolved organic carbon in Adirondack lakes. Ecol Appl 14:839-854

Carlson CA, Ducklow HW (1996) Growth of bacterioplankton and consumption of dissolved organic carbon in the Sargasso Sea. Aquat Microb Ecol 10:69-85

Castillo MM, Kling GW, Allan JD (2003) Bottom-up controls on bacterial production in tropical lowland rivers. Limnol Oceanogr 48:1466-1475

Chantigny MH (2003) Dissolved and water-extractable organic matter in soils: a review on the influence of land use and management practices. Geoderma 113:357-380

Chrzanowski TH, Kyle M, Elser JJ, Sterner RW (1996) Element ratios and growth dynamics of bacteria in an oligotrophic Canadian shield lake. Aquat Microb Ecol 11: $119-125$

Cole JJ, Findlay S, Pace ML (1988) Bacterial production in fresh and saltwater ecosystems - a cross system overview. Mar Ecol Prog Ser 43:1-10

Currie DJ, Kalff J (1984) A comparison of the abilities of freshwater algae and bacteria to acquire and retain phosphorus. Limnol Oceanogr 29:298-310

David MB, Driscoll CT (1984) Aluminum speciation and equilibria in soil solutions of a haplorthod in the Adirondack Mountains (New York, USA). Geoderma 33:297-318

del Giorgio PA, Cole JJ (1998) Bacterial growth efficiency in natural aquatic systems. Annu Rev Ecol Syst 29:503-541

del Giorgio PA, Davis J (2003) Patterns in dissolved organic matter lability and consumption across aquatic ecosystems. In: Findlay SEG, Sinsabaugh RL (eds) Aquatic ecosystems: interactivity of dissolved organic matter. Academic Press, New York, p 400-425

del Giorgio PA, Cole JJ, Cimbleris A (1997) Respiration rates in bacteria exceed phytoplankton production in unproductive aquatic systems. Nature 385:148-151

Dijkstra FA, Smits MM (2002) Tree species effects on calcium cycling: the role of calcium uptake in deep soils. Ecosystems 5:385-398

Dijkstra FA, Geibe C, Holmstrom S, Lundstrom US, van 
Breemen N (2001) The effect of organic acids on base cation leaching from the forest floor under six North American tree species. Eur J Soil Sci 52:205-214

Drakare S (2002) Competition between picoplanktonic cyanobacteria and heterotrophic bacteria along crossed gradients of glucose and phosphate. Microb Ecol 44:327-335

Ducklow HW, Kirchman DL, Anderson TR (2002) The magnitude of spring bacterial production in the North Atlantic Ocean. Limnol Oceanogr 47:1684-1693

Eiler A, Langenheder S, Bertilsson S, Tranvik LJ (2003) Heterotrophic bacterial growth efficiency and community structure at different natural organic carbon concentrations. Appl Environ Microbiol 69:3701-3709

Engelhaupt E, Bianchi TS (2001) Sources and composition of high-molecular-weight dissolved organic carbon in southern Louisiana tidal stream (Bayou Trepagnier). Limnol Oceanogr 46:917-926

Engstrom DR (1987) Influence of vegetation and hydrology on the humus budgets of Labrador lakes. Can J Fish Aquat Sci 44:1306-1314

Farjalla VF, Faria BM, Esteves FA (2002) The relationship between DOC and planktonic bacteria in tropical coastal lagoons. Arch Hydrobiol 156:97-119

Findlay S (2003) Bacterial responses to variation in dissolved organic matter. In: Findlay SEG, Sinsabaugh RL (eds) Aquatic ecosystems: interactivity of dissolved organic matter. Academic Press, New York, p 363-382

Findlay S, Sinsabaugh RL, Fischer DT, Franchini P (1998) Sources of dissolved organic carbon supporting planktonic bacterial production in the tidal freshwater Hudson River. Ecosystems 1:227-239

Finzi AC, Allen AS, DeLucia EH, Ellsworth DS, Schlesinger WH (2001) Forest litter production, chemistry, and decomposition following two years of free air $\mathrm{CO}_{2}$ enrichment. Ecology 82:470-484

Fowler J, Cohen L, Jarvis P (1998) Practical statistics for field biology. Wiley, Chinchester, NY

Frossard E, Brossard M, Hedley MJ, Metherell A (1995) Reactions controlling the cycling of P in soils. In: Tiessen $\mathrm{H}$ (ed) Phosphorus in the global environment: Transfers, cycles, and management. Scope 54. J Wiley, Chichester, p $107-137$

Gaedke U, Hochstadter S, Straile D (2002) Interplay between energy limitation and nutritional deficiency: empirical data and food web models. Ecol Monogr 72:251-270

Garnier J, Benest D (1991) Planktonic bacterial biomass and seasonal pattern of the heterotrophic uptake and respiration of glucose and amino acids in the shallow sandpit Lake of Creteil (Paris suburb, France). Hydrobiologia 209: 191-202

Gergel SE, Turner MG, Kratz TK (1999) Dissolved organic carbon as an indicator of the scale of watershed influence on lakes and rivers. Ecol Appl 9:1377-1390

Goldman JC, Dennett MR (2000) Growth of marine bacteria in batch and continuous culture under carbon and nitrogen limitation. Limnol Oceanogr 45:789-800

Goni MA, Ruttenberg C, Eglington TI (1997) Sources and contributions of terrigenous organic carbon to surface sediments in the Gulf of Mexico. Nature 389:275-278

Gonzalez N, Anadon R, Viesca L (2003) Carbon flux through the microbial community in a temperate sea during summer: role of bacterial metabolism. Aquat Microb Ecol 33: 117-126

Goodale CL, Aber JD, McDowell WH (2000) The long-term effects of disturbance on organic and inorganic nitrogen export in the White Mountains, New Hampshire. Ecosystems 3:433-450
Grieve IC, Marsden RL (2001) Effects of forest cover and topographic factors on TOC and associated metals at various scales in western Scotland. Sci Total Environ 265: $143-151$

Hanson PC, Bade DL, Carpenter SR, Kratz TK (2003) Lake metabolism: relationships with dissolved organic carbon and phosphorus. Limnol Oceanogr 48:1112-1119

Herrera-Silveira JA, Ramírez-Ramírez J (1996) Effects of natural phenolic material (tannin) on phytoplankton growth. Limnol Oceanogr 41:1018-1023

Hinton MJ, Schiff SL, English MC (1997) The significance of storms for the concentration and export of dissolved organic carbon from two Precambrian Shield catchments. Biogeochemistry 36:67-88

Hochstadter S (2000) Seasonal changes of C:P ratios of seston, bacteria, phytoplankton and zooplankton in a deep, mesotrophic lake. Freshw Biol 44:453-463

Hopkinson CS, Fry B, Nolin AL (1997) Stoichiometry of dissolved organic matter dynamics on the continental shelf of the northeastern USA. Cont Shelf Res 17:473-489

Hunt AP, Parry JD, Hamilton-Taylor J (2000) Further evidence of elemental composition as an indicator of the bioavailability of humic substances to bacteria. Limnol Oceanogr 45:237-241

Jansson M, Bergstrom AK, Blomqvist P, Drakare S (2000) Allochthonous organic carbon and phytoplankton/ bacterioplankton production relationships in lakes. Ecology 81:3250-3255

Kalbitz, K, Solinger S, Park JH, Michalzik B, Matzner E (2000) Controls on the dynamics of dissolved organic matter in soils: a review. Soil Sci 165:277-304

Karlsson J, Jansson M, Jonsson A (2002) Similar relationships between pelagic primary and bacterial production in clearwater and humic lakes. Ecology 83:2902-2910

Kirchman DL (1993) Leucine incorporation as a measure of biomass production by heterotrophic bacteria. In: Kemp PF, Sherr BF, Sherr EB, Cole JJ (eds) Handbook of methods in aquatic microbial ecology. Lewis Publishers, Boca Raton, FL, p 509-512

Kirchman DL, Rich JH (1997) Regulation of bacterial growth rates by dissolved organic carbon and temperature in the equatorial Pacific Ocean. Microb Ecol 33:11-20

Kritzberg ES, Cole JJ, Pace ML, Graneli W, Bade DL (2004) Authochthonous versus allochthonous carbon sources of bacteria: results from whole-lake ${ }^{13} \mathrm{C}$ addition experiments. Limnol Oceanogr 49:588-596

Langenheder S, Jürgens K (2001) Regulation of bacterial biomass and community structure by metazoan and protozoan predation. Limnol Oceanogr 46:121-134

LaZerte BD, Scott L (1996) Soil water leachate from two forested catchments on the Precambrian Shield, Ontario. Can J For Res 26:1353-1365

Lennon JT (2004a) Experimental evidence that terrestrial carbon subsidies increase $\mathrm{CO}_{2}$ flux from lake ecosystems. Oecologia 138:584-591

Lennon JT (2004b) The energetic importance of terrestrial carbon in lake ecosystems. PhD thesis, Dartmouth College, Hanover, NH

Likens GE, Bormann HF (1970) Chemical analyses of plant tissues from the Hubbard Brook ecosystem in New Hampshire. Yale University, School of Forestry, New Haven, CT

Lovett GM, Mitchell MJ (2004) Sugar maple and nitrogen cycling in the forests of eastern North America. Front Ecol Environ 2:81-88

Manly BFJ (1994) Multivariate statistical methods: a primer. Chapman \& Hall, London

Marschner B, Kalbitz K (2003) Controls of bioavailability and 
biodegradability of dissolved organic matter in soils. Geoderma 113:211-235

McClain ME, Richey JE, Brandes JA, Pimentel TP (1997) Dissolved organic matter and terrestrial-lotic linkages in the central Amazon basin of Brazil. Global Biogeochem Cycles 11:295-311

McKnight DM, Aiken GR (1998) Sources and age of aquatic humus. In: Hessen DO, Tranvik LJ (eds) Aquatic humic substances: ecology and biogeochemistry. SpringerVerlag, Berlin, p 7-37

McKnight DM, Harnish R, Wershaw RL, Baron JS, Schiff S (1997) Chemical characteristics of particulate, colloidal, and dissolved organic material in Loch Vale Watershed, Rocky Mountain National Park. Biogeochemistry 36: 99-124

McNamara NP, Black HIJ, Beresford NA, Parekh NR (2003) Effects of acute gamma irradiation on chemical, physical and biological properties of soils. Appl Soil Ecol 24: $117-132$

Miller RJ (1986) Beyond ANOVA, basics of applied statistics. Wiley, New York

Minocha R, Long S, Magill AH, Aber J, McDowell WH (2000) Foliar free polyamine and inorganic ion content in relation to soil and soil solution chemistry in two fertilized forest stands at the Harvard Forest, Massachusetts. Plant Soil 222:119-137

Moran MA, Hodson RE (1990) Bacterial production on humic and nonhumic components of dissolved organic carbon. Limnol Oceanogr 35:1744-1756

Moran MA, Hodson RE (1994) Dissolved humic substances of vascular plant-origin in a coastal marine-environment. Limnol Oceanogr 39:762-771

Mulholland PJ (2003) Large-scale patterns in dissolved organic carbon concentrations, flux, and sources. In: Findlay SEG, Sinsabaugh RL (eds) Aquatic ecosystems: interactivity of dissolved organic matter. Academic Press, New York, p 139-160

Münster U (1993) Concentrations and fluxes of organic carbon substrates in the aquatic environment. Antonie Leeuwenhoek 63:243-274

Neff JC, Asner GP (2001) Dissolved organic carbon in terrestrial ecosystems: synthesis and a model. Ecosystems 4: 29-48

Nelson PN, Baldock JA, Oades JM (1993) Concentration and composition of dissolved organic carbon in streams in relation to catchment soil properties. Biogeochemistry 19: $27-50$

Neter J, Kutner M, Nachtsheim CJ, Wasserman W (1996) Applied linear statistical models. Irwin, Chicago, IL

Pace ML, Cole JJ (1996) Regulation of bacteria by resources and predation tested in whole-lake experiments. Limnol Oceanogr 41:1448-1460

Preston CM, Trofymow JA (2000) Variability in litter quality and its relationship to litter decay in Canadian forests. Can J Bot 78:1269-1287

Price ML, Bulter LG (1977) Rapid visual estimation and spectrophotometric determination of tannin content of sorghum grain. J Agric Food Chem 25:1268-1273

Qualls RG, Haines BL (1992) Biodegradability of dissolved organic matter in forest throughfall, soil solution, and stream water. Soil Sci Soc Am J 56:578-586

Quideau SA, Chadwick OA, Benesi A, Graham RC, Anderson MA (2001) A direct link between forest vegetation type and soil organic matter composition. Geoderma 104:41-60

Rasmussen JB, Godbout L, Schallenberg M (1989) The humic content of lake water and its relationship to watershed and lake morphometry. Limnol Oceanogr 34:1336-1343
Raymond PA, Bauer JE (2001) Riverine export of aged terrestrial organic matter to the North Atlantic Ocean. Nature 409:497-500

Rivkin RB, Anderson MR (1997) Inorganic nutrient limitation of oceanic bacterioplankton. Limnol Oceanogr 42:730-740

Roland F, Cole JJ (1999) Regulation of bacterial growth efficiency in a large turbid estuary. Aquat Microb Ecol 20: $31-38$

Russell JB, Cook GM (1995) Energetics of bacterial growthbalance of anabolic and catabolic reactions. Microbiol Rev $59: 48-62$

Sala MM, Peters F, Gasol JM, Pedros-Alio C, Marrase C, Vaque D (2002) Seasonal and spatial variations in the nutrient limitation of bacterioplankton growth in the northwestern Mediterranean. Aquat Microb Ecol 27: $47-56$

SAS (1999) SAS user's guide. Statistics. Version 8. SAS Institute, Cary, NC

Schnitzer M (1982) Organic matter characterization. In: Page AL, Miller RH, Keeney DR (eds) Methods of soil analysis Part 2. Chemical and microbiological properties. American Society of Agronomy, Madison, WI, p 581-593

Sherr EB, Sherr BF (2002) Significance of predation by protists in aquatic microbial food webs. Antonie Leeuwenhoek 81:293-308

Sinsabaugh, RL, Findlay S, Franchini P, Fisher D (1997) Enzymatic analysis of riverine bacterioplankton production. Limnol Oceanogr 42:29-38

Smith EM, Prairie YT (2004) Bacterial metabolism and growth efficiency in lakes: the importance of phosphorus availability. Limnol Oceanogr 49:137-147

Smolander A, Kitunen V (2002) Soil microbial activities and characteristics of dissolved organic $\mathrm{C}$ and $\mathrm{N}$ in relation to tree species. Soil Biol Biochem 34:651-660

Sommer M, Thies H, Kolb E, Bachle H, Stahr K (1997) Biogeochemistry of a cirque-lake landscape: an interdisciplinary study in a catchment of the northern Black Forest, Germany. Water Resour Res 33:2129-2142

Søndergaard M, Middelboe M (1995) A cross-system analysis of labile dissolved organic-carbon. Mar Ecol Prog Ser 118: 283-294

Strobel BW, Hansen HCB, Borggaard OK, Andersen MK, Raulund-Rasmussen K (2001) Composition and reactivity of DOC in forest floor soil solutions in relation to tree species and soil type. Biogeochemistry 56:1-26

Sun L, Perdue EM, Meyer JL, Weis J (1997) Use of elemental composition to predict bioavailability of dissolved organic matter in a Georgia river. Limnol Oceanogr 42:714-721

Templer P, Findlay S, Lovett G (2003) Soil microbial biomass and nitrogen transformations among five tree species of the Catskill Mountains, New York, USA. Soil Biol Biochem 35:607-613

Thoss V, Baird MS, Lock MA, Courty PV (2002) Quantifying the phenolic content of freshwaters using simple assays with different underlying reaction mechanisms. J Environ Monit 4:270-275

Thurman EM (1985) Organic geochemistry of natural waters. Martinus Nijhoff/Junk, Dordrecht

Tranvik LJ (1988) Availability of dissolved organic carbon for planktonic bacteria in oligotrophic lakes of differing humic content. Microb Ecol 16:311-322

Tranvik LJ (1990) Bacterioplankton growth on fractions of dissolved organic carbon of different molecular-weights from humic and clear waters. Appl Environ Microbiol 56: 1672-1677

Vallino JJ, Hopkinson CS, Hobbie JE (1996) Modeling bacterial utilization of dissolved organic matter: optimization 
replaces Monod growth kinetics. Limnol Oceanogr 41: 1591-1609

Vinebrooke RD, Leavitt PR (1998) Direct and interactive effects of allochthonous dissolved organic matter, inorganic nutrients, and ultraviolet radiation on an alpine littoral food web. Limnol Oceanogr 43:1065-1081

Vrede K, Heldal M, Norland S, Bratbak G (2002) Elemental composition $(C, N, P)$ and cell volume of exponentially growing and nutrient-limited bacterioplankton. Appl Environ Microbiol 68:2965-2971

Waiser MJ, Robarts RD (2004) Net heterotrophy in productive prairie wetlands with high DOC concentrations. Aquat Microb Ecol 34:279-290

Editorial responsibility: Robert Sanders,

Philadelphia, Pennsylvania, USA
Walker JM (1996) The bicinchoninic acid (BCA) assay for protein quantitation. In: Walker JM (ed) The protein protocols handbook. Humana Press, Totowa, NJ, p 11-14

Weiss M, Simon M (1999) Consumption of labile dissolved organic matter by limnetic bacterioplankton: the relative significance of amino acids and carbohydrates. Aquat Microb Ecol 17:1-12

Wright RT, Hobbie JE (1965) The uptake of organic solutes in lake water. Limnol Oceanogr 10:22-28

Zsolnay A (1996) Dissolved humus in soil waters. In: Piccolo A (ed) Humic substances in terrestrial ecosystems. Elsevier, Amsterdam, p 171-223

Submitted: October 7, 2004; Accepted: January 3, 2005 Proofs received from author(s): April 26, 2005 